\title{
Determination of porcine gelatin in edible bird's nest by competitive indirect ELISA based on anti-peptide polyclonal antibody
}

\begin{abstract}
Competitive indirect enzyme-linked immunosorbent assay (ELISA) was developed for rapid detection of porcine gelatin in edible bird's nest (EBN). Three ELISAs were developed by using polyclonal rabbit antibodies against porcine species-specific amino acid sequences of collagen Ŭ2 (I) chain (pAb1 and pAb2) and Ŭl (I) chain (pAb3). The limit of detection (IC15) of the three ELISAs was $0.033,0.082$ and $0.052 \varepsilon \mathrm{g} / \mathrm{mL}$ respectively. The median inhibitory concentration (IC50) of pAb1, pAb2 and pAb3 was $0.265,0.394$ and $0.228 \varepsilon \mathrm{g} / \mathrm{mL}$ respectively, as well as able to recognise porcine and bovine gelatins. pAb1 showed slight cross-reactivity with cave nest and egg white, while pAb2 exhibited slight cross-reactivity with blood cave nest and egg white. No cross-reactivity was observed with EBNs and egg white for $\mathrm{pAb3}$. The recoveries of porcine gelatin spiked EBNs were in the range of $62.8 \mathrm{i}$ $125.4 \%$ with intra- and inter-day coefficient of variants (CVs) of $2.9 \ddot{1} 5.4 \%$ and $4.710 .6 \%$ respectively when using pAb3. Taking into account all abovementioned factors, pAb3 appeared sufficient for EBN authentication.
\end{abstract}

Keyword: Edible bird's nest; Gelatin; Polyclonal antibodies; Competitive indirect ELISA 